\title{
On $p$-adic $L$-functions attached to elliptic curves with complex multiplication and the Riemann-Hurwitz genus formula
}

\author{
AKIRA AIBA*
}

\section{§o. Introduction}

Let $K$ be a quadratic imaginary field and let $p$ be a prime number which splits in $K$, say $(p)=\mathfrak{p} \bar{p}$. Let $K_{\infty}$ be the unique $Z_{p}$ extension of $K$ unramified outside $\mathfrak{p}$. Let $F$ be an abelian extension of $K$ of order prime to $p$ and $F_{\infty}=$ $F K_{\infty}$. Let $M_{\infty}$ be the maximal abelian $\mathbf{Z}_{p}$-extension of $F_{\infty}$ unramified outside $p$. Write $X_{\infty}$ for the Galois group of $M_{\infty}$ over $F_{\infty}$, endowed with its natural action of the Galois group $\operatorname{Gal}\left(F_{\infty} / K\right)$. Let $\Gamma=\operatorname{Gal}\left(F_{\infty} / F\right)$. It is well known that $X_{\infty}$ is a finitely generated $Z_{p}[[\Gamma]]$-torsion $\mathbf{Z}_{p}[[\Gamma]]$-module.

In [2] Gillard and in [7] Schneps proved that the $\mu$-invariant of $X_{\infty}$ is 0 inspired by the work of Sinnott [9] in the cyclotomic case. On the other hand in [6] Kida proved an analogue of the Riemann-Hurwitz genus formula by describing the $\lambda$-invariants in cyclotomic case. Kida's formula has three proves: the first one is in Kida [6] using genus theory, the second one is in Iwasawa [5] using Galois cohomology and the third one is in Grass [3,4] and Sinnott [8] using $p$ adic $L$-functions. We study here the elliptic analogue of Kida's formula to get our main theorem (Theorem 6). Our method is followed by the third one especially Sinnott's paper.

\section{§1. Notations}

Let $K$ be an imaginary quadratic field with the integer ring $\circ$. Let $p$ be a rational prime which splits in $K$, say $(p)=p \bar{p}$. Let $K_{\infty}$ be the unique $\mathrm{Z}_{p^{-}}$ extension of $K$ unramified outside $\mathfrak{p}$ and let $K_{n}(n=0,1, \ldots)$ be the unique subfield of $K_{\infty}$ such that $\left[K_{n}: K\right]=p^{n}$. Let $F$ be an abelian extension of $K$ of order prime to $p$. Let $F_{n}=F K_{n}$ and $F_{\infty}=F K_{\infty}$. Let $\Gamma=\operatorname{Gal}\left(F_{\infty} / F\right)$ be the Galois group of $F_{\infty}$ over $F$. Let $M_{\infty, F}$ be the maximal $p$-extension of $F_{\infty}$ unramified outside $p$. Write $X_{\infty, F}=\operatorname{Gal}\left(M_{\infty, F} / F_{\infty}\right)$, endowed with its natural action of $\operatorname{Gal}\left(F_{\infty} / K\right)$. Let $f$ be an integral ideal of $K$ and let $K(f)$ be the ray class field $\bmod f$ of $K$. Let $K\left(f p^{\infty}\right)=\bigcup_{n} K\left(f p^{n}\right)$ and $G(f)=G a l\left(K\left(f p^{\infty}\right) / K\right)$. Let $\chi$ be a finite order character from $\operatorname{Gal}\left(F_{\infty} / K\right)$ to $\mathbf{C}_{p}^{\times}$. Fix a homomorphism

$$
\begin{aligned}
& \kappa: \operatorname{Gal}\left(F_{\infty} / K\right) \simeq \operatorname{Gal}\left(F_{\infty} / K_{\infty}\right) \times \operatorname{Gal}\left(K_{\infty} / K\right) \rightarrow \operatorname{Gal}\left(K_{\infty} / K\right) \simeq\left(1+p \mathbf{Z}_{p}\right) \quad \text { if } \quad p \neq 2 \\
& \simeq\left(1+4 Z_{p}\right) \text { if } p=2
\end{aligned}
$$

Received March 27, 1990.

* Department of Mathematics, Ibaraki University, Mito, Ibaraki 310, Japan. 
Suppose that $f$ divides non $p$-part of the conductor of $\chi$. We may assume that $F$ is contained in $K(\mathfrak{f})$. Let $E$ be an elliptic curve defined over $K(f)$ with fixed Weierstrass model, satisfying the following two conditions. (1) $E$ has complex multiplication by 0 with grössencharacter $\psi$. Thus $\psi$ is a homomorphism from the group of all fractional ideal of $K(f)$ relatively prime to some ideal into $\overline{\mathbf{Q}}$. (2) $\psi(\mathfrak{a})=\kappa\left(\sigma_{N_{K(f) / K^{a}}}\right)$ where $\mathfrak{a}$ is an ideal relatively prime to $\mathfrak{p f}$ and $\sigma_{\mathfrak{a}}=$ $\left(\mathfrak{a}, F_{\infty} / K\right)$ is the Artin symbol of $a$. Let $L$ be the period lattice of $E$. Replacing $E$ by one of its conjugates, if necessary, we assume

$$
L=\Omega \mathfrak{f}, \quad \Omega \in \mathbf{C}^{\times}
$$

Let $\Omega_{p}$ be a $p$-adic period of $E$.(See [1]) Let

$$
L_{\infty, f}\left(\chi \kappa^{-k}, s\right)=\frac{(s+k)}{(2 \pi)^{s+k}} \sum_{(a, f)=1} \frac{\chi \kappa^{-k}\left(\sigma_{a}\right)}{N a^{s}}
$$

where $\sigma_{a}=\left(a, F_{\infty} / K\right)$ be the Artin symbol of $a$ and $\Gamma(s)$ be the gamma function.

We follow the notation of [1] in reviewing the construction of the $p$-adic $L$-function $L_{p, f}(\chi, s)$,

$$
\Omega_{p}^{k} L_{p, f}(\chi, k)=\Omega^{k} G\left(\chi \kappa^{-1}\right)\left(1-\left(\chi^{-1} \kappa^{k}\left(\sigma_{q}\right)\right) / p\right) L_{\infty, f}\left(\chi \kappa^{-k}, 0\right) \quad 1 \leq k \in \mathbf{Z}
$$

Here $G\left(\chi \kappa^{-k}\right) \cdot$ is defined as follows. Let

$$
S=\left\{\gamma \in \operatorname{Gal}\left(K\left(\mathfrak{f p} \mathfrak{p}^{n} \overline{\mathfrak{p}}^{\infty}\right) / K\right)|\quad \gamma|_{\operatorname{Gal}\left(K\left(\mathfrak{f} \bar{p}^{\infty}\right) / K\right)}=\left(\mathfrak{p}^{n}, K\left(f \overline{\mathfrak{p}}^{\infty}\right) / K\right)\right\}
$$

where $n$ is the exact power of $p$ dividing the conductor of $\chi \kappa^{-k}$ and $\zeta_{n}$ is a primitive $p$-th root of unity. Then

$$
G\left(\chi \kappa^{-1}\right)=\frac{\kappa\left(\mathfrak{p}^{n}\right)}{p^{n}} \sum_{\gamma \in S} \chi(\gamma)\left(\zeta_{n}^{\gamma}\right)^{-1}
$$

It is known that there exists a measure $\mu_{f}$ on $G(f)$ such that

$$
L_{p, f}(\chi, s)=\int_{G(f)} \chi^{-1} \kappa^{s}(\sigma) d \mu_{f}
$$

and a power series $G_{p, f}(\chi, T)=\int_{G(f)} \chi^{-1}(\sigma)(1+T)^{\kappa(\sigma)} d \mu_{f}$ such that

$$
L_{p, f}(\chi, s)=G_{p, f}\left(\chi ; u^{s}-1\right)
$$

where $u=\kappa\left(\gamma_{0}\right)$ and $\gamma_{0}$ is a generator of $\operatorname{Gal}\left(K_{\infty} / K\right) \simeq \operatorname{Gal}\left(F_{\infty} / F\right)$. Let $\mathfrak{o}^{\prime}$ be the ring of integers in a finite extension of $\mathbf{Q}_{p}$. Let $f(T)=a_{0}+a_{1} T+$ $a_{2} T^{2}+\ldots \in \Lambda=o^{\prime}[[T]]$ be a non zero power series with coefficients in $o^{\prime}$. Let $\mu(f)=\min \left\{\operatorname{ord}_{p} a_{i}: i \geq 0\right\}$ be the $\mu$-invariant of $f$ and $\lambda(f)=\min \{i \geq 0$ : $\left.\operatorname{ord}_{p} a_{i}=\mu(f)\right\}$ be the $\lambda$-invariant of $f$. 
Two $\Lambda$-modules are said to be psudo-isomorphic if there is a map between them with finite kernel and cokernel. any finitely generated torsion $\Lambda$-module $Y$ is psudo-isomorphic to a module of the form $\bigoplus \Lambda / f_{i} \Lambda$ for certain $f_{i} \in \Lambda$, and the characteristic power series $\left(\prod f_{i}\right)$ is a well-defined invariant of $Y$ which we will denote by $\operatorname{char}(Y)$. It is well known that $X_{\infty, F}$ is a finitely generated torsion $\Lambda$-module, with the action $T x=\left(\gamma_{0}-1\right) x$. Let $\mu_{F}=\mu\left(\operatorname{char}\left(X_{\infty, F}\right)\right)$ and let $\lambda_{F}=\lambda\left(\operatorname{char}\left(X_{\infty, F}\right)\right)$. Let $\mu_{f}(\chi)=\mu\left(G_{p, f}(\chi, T)\right)$ and let $\lambda_{f}(\chi)=\lambda\left(G_{p, f}(\chi, T)\right)$.

If $f$ is the non $p$-part of the conductor $\chi$, we omit the subscript $f$ from our notations: thus $L_{p}(\chi, s), G_{p}(\chi, s), \mu(\chi), \lambda(\chi)$. If $G$ is a group $\hat{G}$ denotes the character group of $G$.

$\S 2$.

We use the next two important lemmas.

LEMMA 1.

$$
\mu_{F}=0
$$

Proof. See Gillard [2] or Schneps [7]

The next lemma is a link between $\mathbf{Z}_{p}$-extension and $L$-function.

LEMMA 2.

$$
\begin{gathered}
\mu_{F}=\sum_{\operatorname{Gal}\left(\widehat{\left.F_{\infty} / K_{\infty}\right)}\right.} \mu(\chi) \\
\lambda_{F}=\sum_{\chi \in G a l\left(\widehat{\left.F_{\infty} / K_{\infty}\right)}\right.} \lambda(\chi)+1
\end{gathered}
$$

Proof. See de Shalit[1]

The next lemma gives some information on $\lambda_{f}(\chi)$ when $f$ is varied.

LEMмA 3. Let $\chi$ be a finite order character of $G a l(F / K)$. Let $\mathfrak{f}$ and $\mathfrak{f}^{\prime}$ be integral ideals of $K$ which are divisible by the non-p-part of the conductor of $\chi$, and suppose that $f$ is divisible by $\mathfrak{f}^{\prime}$. Then

$$
\lambda_{f^{\prime}}(\chi)=\lambda_{f}(\chi)+\sum g(q)
$$

where the summation is taken over primes $q$ which divides $\mathfrak{f}^{\prime} f^{-1}$ in $K$ such that $\chi\left(\sigma_{q}\right)$ has $p$-power order and $g(q)$ denotes the number of places of $K_{\infty}$ lying above $\mathbf{q}$.

Proof. Define a $p$-adic integer $t(q)$ by $\sigma_{q} \equiv \gamma_{0}^{t(q)} \bmod \operatorname{Gal}(F / K)$. where $\gamma_{0}$ is a generator of $\operatorname{Gal}\left(K_{\infty} / K\right)$. Write

$$
-t(\mathfrak{q})=p^{a} u \quad a \geq 0, \quad u \in \mathbf{Z}_{p}^{\times}
$$


From the definition,

$$
\begin{gathered}
L_{p, f^{\prime}}(\chi, s)=L_{p, f}(\chi, s) \prod\left(1-\chi \kappa^{-s}(\mathfrak{q})\right) \\
G_{p, f^{\prime}}(\chi, T)=G_{p, f}(\chi, T) \prod E_{q}(T)
\end{gathered}
$$

where the product is taken over primes $q$ which divide $f^{\prime} f^{-1}$ in $K$ and

$$
E_{\mathrm{q}}(T)=\left(1-\chi\left(\sigma_{q}\right)(1+T)^{-t(q)}\right)
$$

Then

$$
\begin{aligned}
E_{\mathfrak{q}}(T) & \equiv 1-\chi\left(\sigma_{q}\right)\left(1+T^{p^{a}}\right)^{u} \quad \bmod \quad p \mathfrak{o}^{\prime}[[T]] \\
& \equiv 1-\chi\left(\sigma_{\mathfrak{q}}\right)-\chi\left(\sigma_{\mathfrak{q}}\right) u T^{p^{a}} \quad \bmod \quad\left(p, T^{p^{a}+1}\right) \mathfrak{o}^{\prime}[[T]]
\end{aligned}
$$

It follows that

$$
\begin{aligned}
\mu\left(E_{\mathrm{q}}(T)\right) & =0 \\
\lambda\left(E_{\mathrm{q}}(T)\right) & =p \quad \text { if } \chi\left(\sigma_{\mathrm{q}}\right) \text { is a } p \text {-power root of unity } \\
& =0 \text { otherwise }
\end{aligned}
$$

Let $D_{q}$ (resp. $I_{q}$ ) be the decomposition(resp.inertia) group of $\mathfrak{q}$ for the extension $K_{\infty} / K$. Then $D_{q} / I_{q}$ is generated by

$$
\gamma_{0} \bmod \operatorname{Gal}(F / K)
$$

It follows that $g(q)$ is finite and equal to $p^{a}$ q.e.d.

The next lemma gives some information on $\lambda_{f}(\chi)$ when $\chi$ is varied.

LEMMA 4. Let $\chi$ be a finite order character of $G a l\left(F_{\infty} / K\right)$ and let $\psi$ be a finite order character of $\operatorname{Gal}\left(F_{\infty} / K\right)$ of $p$-power order. Then

$$
\lambda_{f}(\chi)=\lambda_{f}(\chi \psi)
$$

Proof. Let $\mathfrak{o}_{\chi, \psi}$ be the ring of integers in a finite extension of $\mathbf{Q}_{p}$ containing the values of both $\chi$ and $\psi$ and let $\pi$ be a local parameter in ${ }^{0} \chi, \psi$

Then since $\psi$ has a $p$-power order,

$$
G_{p, f}(\chi \psi, T)-G_{p, f}(\chi, T)=\int\left(\psi^{-1}(\sigma)-1\right) \chi^{-1}(\sigma)(1+T)^{\kappa(\sigma)} d \mu_{f} \in \pi \mathbf{o}_{\chi, \psi}[[T]]
$$

Since $\mu_{f}(\chi)=\mu_{\mathfrak{f}}(\chi \psi)=0$, the result is obtained.

q.e.d.

LEMMA5. Let $\chi$ be a finite order character of $\operatorname{Gal}\left(F_{\infty} / K\right)$ and let $\psi$ be a finite order character of $G a l\left(F_{\infty} / K\right)$ of p-power order. Suppose that the order 
of $\chi$ be prime to $p$. Let $L$ be the extension of $K$. corresponding to $\chi$ and let $L_{\infty}=L K_{\infty}$. Then

$$
\lambda(\chi \psi)=\lambda(\chi)+N
$$

where $N$ is the number of places $v$ on $K_{\infty}$ such that (1) $v$ doesn't lie above $p$ and $\left.v\right|_{K}$ is ramified for $\psi$. (2) $v$ splits completely in $L_{\infty}$.

Proof. Let $f\left(\right.$ resp. $\left.f^{\prime}\right)$ be the non $p$-part of the conductor of $\chi(\operatorname{resp} . \chi \psi)$. Since $\chi$ and $\psi$ have relatively prime orders, $f^{\prime}$ is divisible by $f$. By Lemma 3 and Lemma 4 ,

$$
\lambda(\chi \psi)=\lambda_{f^{\prime}}(\chi \psi)=\lambda_{f^{\prime}}(\chi)=\lambda(\chi)+M
$$

where $M=\sum g(q)$, the summation taken over places $q$, which divides $\mathfrak{f}^{\prime} \mathfrak{f}^{-1}$ for which $\chi\left(\sigma_{q}\right)$ has $p$-power.

Since $\chi$ has order prime to $p, \chi\left(\sigma_{q}\right)$ is $p$-power order if and only if $\chi\left(\sigma_{q}\right)=1$, that is $q$ splits completely in $L$. Then from the definition of $g(q), M$ is the number of places $v$ on $K_{\infty}$ which split completely in $L_{\infty}$ and $\left.v\right|_{K}=q$ is prime number which divides $f^{\prime} f^{-1}$. Such $v$ satisfy (1) and (2). Conversely in $L$ and from the condition that order of $\chi$ is prime to order of $\psi,\left.v\right|_{K}$ divides $f^{\prime} f^{-1} \quad$ q.e.d.

The next result is the main theorem.

THEOREM 6. Notations are as usual as in $\S 1$. Let $H \supset F \supset K$ be a tower of abelian extensions such that $\mathrm{Gal}(H / F)$ is $p$-power order and the order of $G a l(F / K)$ is prime to $p$. Let $\lambda_{F}$ and $\lambda_{H}$ be the $\lambda$-invariant of $X_{\infty, F}$ and $X_{\infty, H}$, respectively. Then

$$
\lambda_{H}-1=\left[H_{\infty}: F_{\infty}\right]\left(\lambda_{F}-1\right)+\sum_{w}(e(w / v)-1)
$$

where the summation is taken over all places $w$ on $H_{\infty}$ which don't lie above $p$ and $v=\left.w\right|_{F_{\infty}}$ and $e(w / v)$ denotes the ramification index of $w$ over $v$.

Proof. If $H \cap F_{\infty}$ doesn't contain $F$, take the subfield $H^{\prime \prime}$ of $H$ containing $F$ such that $H^{\prime \prime} \cap F_{\infty}=F, H_{\infty}^{\prime \prime}=H_{\infty}$ and we may assume $H \cap F_{\infty}=F$.

We prove only the case when $[H: F]=p$ and $[F: K]=q(p, q$ : prime numbers of $p \neq q$ ). The other cases are the same way to prove.

Difine $H^{\prime}$ the subfield of $H$ such that $H \cong H^{\prime} \times F$. We have a factorization

$$
\prod_{\theta \in G a l(H / K)} L_{p}(\theta, s)=\prod_{\psi \in G a l\left(H^{\prime} / K\right)} \prod_{\chi \in G a \hat{l}(F / K)} L_{p}(\chi \psi, s)
$$

and

$$
G_{p}(\theta, T)=\prod \prod G_{p}(\chi \psi, T)
$$

then

$$
\sum \lambda(\theta)=\sum \sum \lambda(\chi \psi)
$$

Let $N$ be the number of places $v$ of $K_{\infty}$ such that (1) $v$ doesn't lie above $p$ and $\left.v\right|_{K}$ is ramified for $\psi$, where $\psi$ denotes a non-trivial character of $\operatorname{Gal}\left(H^{\prime} / K\right)$. 
Let $N^{\prime}$ be the number of places $v$ of $K$ such that (1) and (2) $v$ splits completely in $F$. Then from the lemmas,

$$
\begin{aligned}
\sum_{\theta \in G a \widehat{l(H / K)}} \lambda(\theta) & =\sum_{1 \neq \chi \in G a \widehat{l(F / K)}}\left(p \lambda(\chi)+(p-1) N^{\prime}\right)+p \lambda(1)+(p-1) N \\
& =p \sum_{\chi \in G a \widehat{l(F / K)}} \lambda(\chi)+(p-1)\left((q-1) N^{\prime}+N\right) \\
& =p \sum \lambda(\chi)+\sum_{w}(e(w / v)-1)
\end{aligned}
$$

where the second summation is taken over the places $w$ of $H_{\infty}$ which don't lie above $p$. From Lemma 2, we obtain the result.

q.e.d.

\section{References}

[1] E.de Schalit, Iwasawa theory of elliptic curves with complex multiplication, Academic press, New York-London, 1987.

[2] R.Gillard, Fonction $L$ p-adiques des corps quadratiques imaginaires et de leurs extensions abéliennes, J. Reine Angew. Math., 358 (1985), 76-91.

[3] G. Grass, Sur la construction des fonctions $L$ p-adiques abélienne, Seminaire DelangePisot-Poitou (Théorie des nombres) 22 (1978/79).

[4] G.Grass, Sur les invariants d'Iwasawa des corps abéliens, Pub. Math. de la Fac. des Sci. de Besançon' (1978/79).

[5] Iwasawa K., Riemann-Hurwitz formula and p-adic Galois representations for number fields, Tōhoku Math. J.(second series) 33(2) (1981), 263-288.

[6] Kida Y., l-extensions of CM-fields and cyclotomic invariants, J. Number Theory 12 (1980), 519-528.

[7] L. Schneps, On the $\mu$-invariant of $p$-adic $L$-functions attached to elliptic curves with complex multiplication, J. Number Theory 25 (1987), 20-33.

[8] W.Sinnott, On p-adic $L$-functions and the Riemann-Hurwitz genus formula, Composito Math. 53 (1984), 3-17.

[9] W.Sinnott, On the $\mu$-invariant of a rational function, Invent. Math. 75 (1984), 273-283. 